\begin{tabular}{|c|c|}
\hline Title & Interwall screening and excitons in double wall carbon nanotubes \\
\hline Author(s) & Tomio, Y uh; Suzuura, Hidekatsu; A ndo, Tsuneya \\
\hline Citation & $\begin{array}{l}\text { Physical Review B, 85(8), 085411 } \\
\text { https://doi.org/10.1103PPhysRevB.85.085411 }\end{array}$ \\
\hline Issue Date & 2012-02-15 \\
\hline Doc URL & http:/hdl.handle.net/2115/48523 \\
\hline Rights & @2012 A merican Phy sical Society \\
\hline Type & article \\
\hline File Information & PRB85-8_085411.pdf \\
\hline
\end{tabular}

Instructions for use 


\title{
Interwall screening and excitons in double-wall carbon nanotubes
}

\author{
Yuh Tomio, ${ }^{1, *}$ Hidekatsu Suzuura, ${ }^{1}$ and Tsuneya Ando ${ }^{2}$ \\ ${ }^{1}$ Division of Applied Physics, Graduate School of Engineering, Hokkaido University, Sapporo 060-8628, Japan \\ ${ }^{2}$ Department of Physics, Tokyo Institute of Technology, 2-12-1 Ookayama, Meguro-ku, Tokyo 152-8551, Japan
}

(Received 2 December 2011; published 7 February 2012)

\begin{abstract}
Exciton properties of double-wall carbon nanotubes are studied in the static screened Hartree-Fock approximation within a $\boldsymbol{k} \cdot \boldsymbol{p}$ scheme. The intrawall electron-hole interaction is largely suppressed by interwall screening effects. The suppression is sensitive to the effective interwall distance between the inner and outer tubes and reduces the exciton binding energy as well as the band gap. As a result, the exciton energy levels are redshifted from those in the single-wall tube with the same diameter. The energy shift of the ground exciton has little dependence on the tube diameter, in contrast to that of the excited excitons. In the case of a metallic outer and inner tube, excited exciton states in the semiconducting tube disappear due to strong screening.
\end{abstract}

DOI: 10.1103/PhysRevB.85.085411

PACS number(s): 78.67.Ch, 73.22.Lp, 71.35.Cc

\section{INTRODUCTION}

Carbon nanotubes have attracted much attentions for their unique physical properties ${ }^{1-4}$ since their discovery, and, therefore, many experimental and theoretical studies have been dedicated from viewpoints of both fundamental physics and applications. Their optical properties are particularly interesting because exciton states play a dominant role $\mathrm{e}^{5-12}$ and their binding energies are unusually larger than those in bulk semiconductor systems, originating from the onedimensionality and the strong Coulomb interaction. The purpose of this paper is to study effects of interwall screening on excitons in double-wall nanotubes.

The screening effect is known to change exciton energies unconventionally in nanotubes. In fact, recent theoretical study suggested that antiscreening is responsible for the large binding energies of excited exciton states in semiconducting single-wall nanotubes. ${ }^{13}$ In experiments, the photoluminescence studies $^{14,15}$ of double-wall nanotubes have reported that the energies of the optically active exciton states of the inner tubes are redshifted from those of single-wall tubes with the same chiral indices, where the effective dielectric screening is expected to be modified by the presence of the surrounding outer tube, i.e., environmental effects. ${ }^{16-19}$ Double-wall tubes are the simplest systems of multiwall carbon nanotubes and thus are suited for studying the interactions between different walls. $^{20-28}$

In this work we examine effects of screening by the interwall Coulomb interaction on excitons and optical spectra in double-wall tubes based on the effective-mass theory and a static screened Hartree-Fock approximation. We shall focus on excitons in a semiconducting nanotube surrounded by or containing another coaxial semiconducting or metallic nanotube.

The paper is organized as follows: In Sec. II, we briefly review an effective-mass description of energy bands of carbon nanotubes, the Coulomb potential and dielectric function in multiwall nanotubes, and a method of calculation of exciton states. Numerical results are presented in Sec. III, which discusses how exciton properties depend on the interwall distance and semiconducting or metallic screening. The dependence on the diameter is discussed in Sec. IV. The results are discussed and a short summary is given in Sec. V.

\section{FORMULATION}

In a graphene sheet the conduction and valence bands consisting of $\pi$ orbitals cross at $K$ and $K^{\prime}$ points, where the Fermi level is located. Electronic states of the $\pi$ bands near a $K$ point are described by the $\boldsymbol{k} \cdot \boldsymbol{p}$ equation, ${ }^{2,29-34}$

$$
\gamma(\boldsymbol{\sigma} \cdot \hat{\boldsymbol{k}}) \boldsymbol{F}(\boldsymbol{r})=\varepsilon \boldsymbol{F}(\boldsymbol{r})
$$

where $\gamma$ is a band parameter, related to nearest-neighbor hopping integral $\gamma_{0}$ through $\gamma=(\sqrt{3} / 2) a \gamma_{0}$ with the lattice constant $a=0.246 \mathrm{~nm} ; \sigma=\left(\sigma_{x}, \sigma_{y}\right)$ is the Pauli spin matrix; and $\hat{\boldsymbol{k}}=\left(\hat{k}_{x}, \hat{k}_{y}\right)=-i \nabla$ is a wave-vector operator.

The structure of a nanotube is specified by a chiral vector $\boldsymbol{L}$ corresponding to the circumference. For tubes with a sufficiently large diameter, the energy bands are obtained by imposing the boundary conditions around the circumference direction,

$$
\boldsymbol{F}(\boldsymbol{r}+\boldsymbol{L})=\boldsymbol{F}(\boldsymbol{r}) \exp \left(-\frac{2 \pi i v}{3}\right)
$$

where $v$ is an integer $(v=0$ or \pm 1$)$ determined by the structure. For $v=0$, the nanotube becomes a metal. For $v= \pm 1$, the nanotube becomes a semiconductor with nonzero gap at the Fermi level.

The energy bands are specified by $(s, n, k)$, where $s=+$ for the conduction and - for the valence band, respectively, and $n$ is an integer corresponding to the discrete wave vector along the circumference direction ( $x$ axis) and the wave vector $k$ in the tube axis direction ( $y$ axis).

Consider a multiwall carbon nanotube consisting of cylinders with radius $R_{j}$ (tube $j$ ) with $j=1,2, \ldots, M$, as illustrated in Fig. 1. The lattice structure of adjacent walls is incommensurate in usual multiwall nanotubes. ${ }^{35,36}$ In such incommensurate cases, interwall electron hopping is negligibly small due to cancellation of interwall coupling at different sites in the absence of disorder. ${ }^{4,37-39}$ In our analysis, therefore, interwall hopping is completely neglected.

Then, the wave function in multiwall nanotubes can be expressed by that in each wall. According to the boundary condition (2), the wave function for a band associated with the 


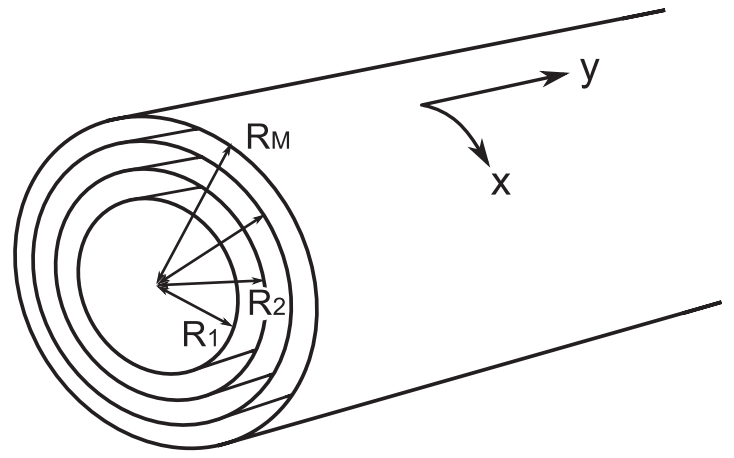

FIG. 1. A schematic illustration of a multiwall carbon nanotube with $M$ walls where $R_{j}$ is the radius of the $j$ th wall.

$K$ point in the tube with radius $R_{j}$ is written as

$$
\boldsymbol{F}_{s n k}^{R_{j}}(\boldsymbol{r})=\frac{1}{\sqrt{A L_{j}}} \exp \left[i \kappa_{v_{j}}(n) x+i k y\right] \boldsymbol{F}_{s n k}^{v_{j}}
$$

where $A$ is the length of the nanotube, the circumference length $L_{j}=2 \pi R_{j}, v_{j}=0, \pm 1$, and

$$
\begin{gathered}
\boldsymbol{F}_{s n k}^{v_{j}}=\frac{1}{\sqrt{2}}\left[\begin{array}{c}
b_{v_{j}}(n, k) \\
s
\end{array}\right], \\
\kappa_{v_{j}}(n)=\frac{2 \pi}{L_{j}}\left(n-\frac{v_{j}}{3}\right), \\
b_{v_{j}}(n, k)=\frac{\kappa_{v_{j}}(n)-i k}{\sqrt{\kappa_{v_{j}}(n)^{2}+k^{2}}} .
\end{gathered}
$$

The corresponding energy is given by

$$
\varepsilon_{j, s, n}^{K}(k)=s \gamma \sqrt{\kappa_{\nu_{j}}(n)^{2}+k^{2}} .
$$

For the $K^{\prime}$ point, the $\boldsymbol{k} \cdot \boldsymbol{p}$ Hamiltonian is obtained by replacing $\hat{k}_{y}$ with $-\hat{k}_{y}$ in Eq. (1) and the boundary condition [Eq. (2)] is obtained by replacing $v$ with $-v$. Therefore, the energy band is given by Eq. (7) in which $\kappa_{v_{j}}(n)$ is replaced with $\kappa_{-v_{j}}(n)$ and the wave function is given by Eq. (3), in which $b_{v_{j}}(n, k)$ is replaced with $b_{-v_{j}}(n, k)^{*}$. This corresponds to the fact that the $K$ and $K^{\prime}$ points are related to each other via the time-reversal operation $T$, given by $\boldsymbol{F}_{K}^{T}=e^{-i \psi} \sigma_{z} \boldsymbol{F}_{K^{\prime}}^{*}$ and $\boldsymbol{F}_{K^{\prime}}^{T}=e^{-i \psi} \sigma_{z} \boldsymbol{F}_{K}^{*}$, with the Pauli matrix $\sigma_{z}$ and an arbitrary phase $\psi \cdot{ }^{40,41}$ Because Coulomb matrix elements of intervalley scattering between the $K$ and $K^{\prime}$ points are much smaller than those of intravalley scattering, the bands associated with the $K$ and $K^{\prime}$ points are separated from each other and degenerate due to the time reversal symmetry in the absence of a magnetic field.

The Coulomb interaction between an electron at $\boldsymbol{r}=(x, y)$ on a cylinder surface with radius $R_{i}$ and another at $\boldsymbol{r}^{\prime}=\left(x^{\prime}, y^{\prime}\right)$ on a cylinder surface with radius $R_{j}$ is given by

$$
v_{i j}\left(\theta-\theta^{\prime}, y-y^{\prime}\right)=\sum_{m} \int \frac{d q}{2 \pi} e^{i m\left(\theta-\theta^{\prime}\right)} e^{i q\left(y-y^{\prime}\right)} V_{i j}^{0}(m, q),
$$

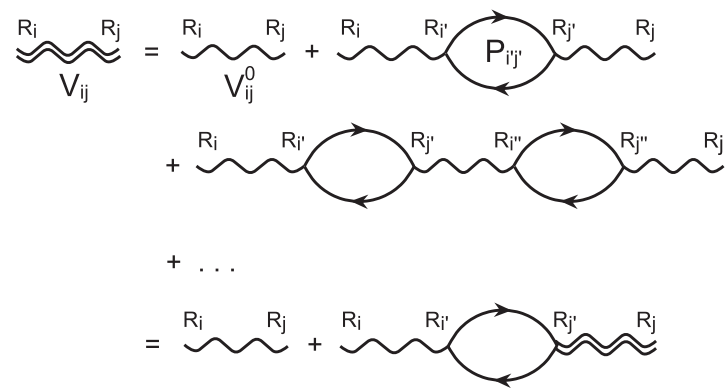

FIG. 2. The diagrammatic representation of the effective Coulomb interaction in multi wall nanotubes.

where $\theta=x / R_{i}, \theta^{\prime}=x^{\prime} / R_{j}, m$ is an integer, and $q$ is the wave number in the axis direction. The Fourier coefficient in Eq. (8) is written as ${ }^{42-44}$

$$
V_{i j}^{0}(m, q)=2 e^{2} I_{|m|}\left(|q| R_{\min }\right) K_{|m|}\left(|q| R_{\max }\right),
$$

with

$$
R_{\min }=\min \left(R_{i}, R_{j}\right), \quad R_{\max }=\max \left(R_{i}, R_{j}\right),
$$

where $I_{m}(t)$ and $K_{m}(t)$ are the modified Bessel function of the first and the second kinds, respectively.

In the random-phase approximation or in the self-consistent field approximation, the screening of the Coulomb interaction contains a series of bubble diagrams as shown in Fig. 2. Therefore, within the static approximation, the effective Coulomb interaction between two electrons on cylinders with radius $R_{i}$ and $R_{j}$ can be expressed by a matrix,

$$
\hat{V}(m, q)=\left(\begin{array}{cccc}
V_{11} & V_{12} & \ldots & V_{1 M} \\
V_{21} & V_{22} & \ldots & V_{2 M} \\
\vdots & \vdots & \ddots & \vdots \\
V_{M 1} & V_{M 2} & \ldots & V_{M M}
\end{array}\right),
$$

while the bare Coulomb interaction is given by

$$
\hat{V}^{0}(m, q)=\left(\begin{array}{cccc}
V_{11}^{0} & V_{12}^{0} & \ldots & V_{1 M}^{0} \\
V_{21}^{0} & V_{22}^{0} & \ldots & V_{2 M}^{0} \\
\vdots & \vdots & \ddots & \vdots \\
V_{M 1}^{0} & V_{M 2}^{0} & \ldots & V_{M M}^{0}
\end{array}\right),
$$

and they satisfy

$$
\hat{V}(m, q)=\hat{V}^{0}(m, q)-\hat{V}^{0}(m, q) \hat{P}(m, q) \hat{V}(m, q),
$$

where the matrix consisting of the polarization function is given by

$$
\hat{P}(m, q)=\left(\begin{array}{cccc}
P_{1} & 0 & \ldots & 0 \\
0 & P_{2} & \ldots & 0 \\
\vdots & \vdots & \ddots & \vdots \\
0 & 0 & \ldots & P_{M}
\end{array}\right) .
$$

Here, $P_{j}(m, q)$ is the polarization function of the cylinder with radius $R_{j}$, corresponding to the electron-hole excitation within the same wall. We then have

$$
\hat{V}(m, q)=\left[1+\hat{V}^{0}(m, q) \hat{P}(m, q)\right]^{-1} \hat{V}^{0}(m, q) .
$$


The off-diagonal elements of $\hat{P}(m, q)$, corresponding to the electron-hole excitation between different walls, can be omitted because the interwall hopping can safely be neglected in usual incommensurate cases, as mentioned above.,37-39 Even for some commensurate cases, such as armchair doublewall nanotubes with significant interwall hoppings, their contributions to the polarization function have been shown to be quite small in tight-binding calculations. ${ }^{45}$

The polarization function $P_{j}(m, q)$ of tube $j$ can be divided into two contributions,

$$
P_{j}(m, q)=\Pi_{j}(m, q)+\Pi_{j}^{\prime}(m, q),
$$

where $\Pi_{j}(m, q)$ represents contributions of electrons in the vicinity of the $K$ and $K^{\prime}$ points and $\Pi_{j}^{\prime}(m, q)$ represents those in the $\pi$ bands away from the $K$ and $K^{\prime}$ points, in the $\sigma$ bands, and in the core states. In the present study, we replace $\Pi_{j}^{\prime}(m, q)$ with a phenomenological dielectric constant $\kappa$ in such a way that

$$
V_{j j}^{0}(m, q) \Pi_{j}^{\prime}(m, q) \simeq \kappa-1 .
$$

Then, if effects of polarization in tube $j$ are included and those of other tubes are neglected, the effective dielectric function becomes

$$
\epsilon_{j}(m, q)=\kappa+V_{j j}^{0}(m, q) \Pi_{j}(m, q) .
$$

This shows that $\kappa$ is formerly regarded as a background dielectric constant for a single-wall nanotube. In multiwall nanotubes, however, such a simple replacement by a background dielectric constant becomes no longer valid because of the strong $q$ dependence of interwall Coulomb potential even in the approximation of Eq. (17), as will be shown in the next section.

Explicitly, we have

$$
\Pi_{j}(m, q)=\Pi_{j}^{K}(m, q)+\Pi_{j}^{K^{\prime}}(m, q),
$$

with

$$
\begin{aligned}
\Pi_{j}^{K}(m, q)= & \frac{2 g_{s}}{A} \sum_{n} \sum_{k}\left|\boldsymbol{F}_{-, n, k}^{v_{j} \dagger} \cdot \boldsymbol{F}_{+, m+n, k+q}^{v_{j}}\right|^{2} \\
& \times \frac{g_{0}\left[\varepsilon_{j,+, m+n}^{K}(k+q)\right] g_{0}\left[\varepsilon_{j,-, n}^{K}(k)\right]}{\varepsilon_{j,+, m+n}^{K}(k+q)-\varepsilon_{j,-, n}^{K}(k)},
\end{aligned}
$$

where $g_{s}=2$ is the spin degeneracy and $g_{0}(\varepsilon)$ is a cutoff function, defined by

$$
g_{0}(\varepsilon)=\frac{\varepsilon_{c}^{\alpha_{c}}}{|\varepsilon|^{\alpha_{c}}+\varepsilon_{c}^{\alpha_{c}}} .
$$

The cutoff function containing two parameters $\alpha_{c}$ and $\varepsilon_{c}$ should be chosen in such a way that we include only the contributions from states in the vicinity of the Fermi level. The appropriate value of $\varepsilon_{c}$ is about the half of the $\pi$ band width $\sim 3 \gamma_{0}$ and the results are essentially independent of $\alpha_{c}$ when $\alpha_{c} \geqslant 2$. The polarization near the $K^{\prime}$ point, $\Pi_{j}^{K^{\prime}}(m, q)$, is given by Eq. (20) with replacements $v_{j} \rightarrow-v_{j}$ and $\varepsilon_{j, s, n}^{K}(k) \rightarrow \varepsilon_{j, s, n}^{K^{\prime}}(k)$.

The exciton state for an electron in the $K$ valley and a hole in the $K$ valley of the tube with radius $R_{j}$ is expressed by

$$
|u ; j\rangle=\sum_{n, k} \psi_{j, n}^{u}(k) c_{j,+, n, k}^{K \dagger} c_{j,-, n, k}^{K}|g\rangle,
$$

where $|g\rangle$ is the ground-state wave function and $c_{j, s, n, k}^{K}$ and $c_{j, s, n, k}^{K \dagger}$ are the annihilation and creation operators, respectively, for states specified by $(s, n, k)$ in the $K$ valley of tube with radius $R_{j}$. The exciton wave function $\psi_{j, n}^{u}(k)$ satisfies the BetheSalpeter equation,

$$
\begin{aligned}
E_{j}^{u} \psi_{j, n}^{u}(k)= & {\left[\tilde{\varepsilon}_{j,+, n}^{K}(k)-\tilde{\varepsilon}_{j,-, n}^{K}(k)\right] \psi_{j, n}^{u}(k) } \\
& -\sum_{m, q} V_{(+, n, k ;+, m, k+q)(-, m, k+q ;-, n, k)}^{j j} \psi_{j, m}^{u}(k+q),
\end{aligned}
$$

with

$$
\begin{aligned}
& V_{\left(s_{1}, n, k ; s_{2}, m, k+q\right)\left(s_{3}, m, k+q ; s_{4}, n, k\right)}^{j j} \\
& \quad=\frac{1}{A} V_{j j}(n-m, q)\left(\boldsymbol{F}_{s_{1}, n, k}^{v_{j} \dagger} \cdot \boldsymbol{F}_{s_{2}, m, k+q}^{v_{j}}\right)\left(\boldsymbol{F}_{s_{3}, m, k+q}^{v_{j} \dagger} \cdot \boldsymbol{F}_{s_{4}, n, k}^{v_{j}}\right),
\end{aligned}
$$

and

$$
\tilde{\varepsilon}_{j, s, n}^{K}(k)=\varepsilon_{j, s, n}^{K}(k)+\Sigma_{j, s, n}^{K}(k) .
$$

The self-energy is given by

$$
\begin{aligned}
& \Sigma_{j, s, n}^{K}(k) \\
& \quad=-\sum_{m, q} V_{(s, n, k ;-, m, k+q)(-, m, k+q ; s, n, k)}^{j j} g_{0}\left[\varepsilon_{j, s, m}^{K}(k+q)\right],
\end{aligned}
$$

in the screened Hartree-Fock approximation.

The optical absorption is described by the dynamical conductivity. For polarization parallel to the tube axis, the dynamical conductivity of tube $j$ is calculated using the Kubo formula as

$$
\sigma_{j}(\omega)=\frac{g_{s} \hbar e^{2}}{A L_{j}} \sum_{K K^{\prime}} \sum_{u} \frac{-2 i \hbar \omega\left|\left\langle u ; j\left|v_{y}\right| g\right\rangle\right|^{2}}{E_{j}^{u}\left(E_{j}^{u 2}-\hbar^{2} \omega^{2}-2 i \hbar \omega \Gamma\right)},
$$

where the velocity operator in the tube axis direction is given by $v_{y}=(\gamma / \hbar) \sigma_{y}$ for the $K$ point and $\Gamma$ is a phenomenological broadening parameter. The matrix element is given by

$$
\begin{aligned}
& \left\langle u ; j\left|v_{y}\right| g\right\rangle=\sum_{n} \sum_{k}\left(v_{y}\right)_{j, n, k} \psi_{j, n}^{u}(k)^{*}, \\
& \left(v_{y}\right)_{j, n, k}=i \frac{\gamma}{2 \hbar}\left[b_{v_{j}}(n, k)^{*}+b_{v_{j}}(n, k)\right],
\end{aligned}
$$

and for the $K^{\prime}$ point it is given by complex conjugate of Eq. (29) with $v_{j} \rightarrow-v_{j}$. The intensity of the absorption is characterized by a dimensionless oscillator strength

$$
f_{j}^{u}=2 m_{j}^{*} \frac{\left|\left\langle u ; j\left|v_{y}\right| g\right\rangle\right|^{2}}{E_{j}^{u}},
$$

where $m_{j}^{*}$ is chosen as the effective mass of the bottom of the lowest conduction band in the tube with radius $R_{j}$, i.e., $m_{j}^{*}=2 \pi \hbar^{2} /\left(3 \gamma L_{j}\right)$.

As long as the absorption in each tube is sufficiently small, the total absorption is proportional to $\sum_{j}\left(2 \pi R_{j}\right) \operatorname{Re} \sigma_{j}(\omega)$. Thus, we shall define the effective dynamical conductivity as the weighted sum of that of each tube, i.e.,

$$
\sigma(\omega)=\bar{R}^{-1} \sum_{j=1}^{M} R_{j} \sigma_{j}(\omega), \quad \bar{R}=\frac{1}{M} \sum_{j=1}^{M} R_{j} .
$$


This is valid when the electric field of light at each tube is nearly the same as the outside field.

In a single-wall nanotube with diameter $R$, the effective strength of the Coulomb interaction is specified by dimensionless interaction parameter $\left(e^{2} / \kappa L\right) /(2 \pi \gamma / L)$ with $L=2 \pi R$. It is estimated as $\left(e^{2} / \kappa L\right) /(2 \pi \gamma / L) \approx 0.4 / \kappa$ for $\gamma_{0}=2.7 \mathrm{eV}$. In bulk graphite we have $\kappa \approx 2.5,{ }^{46}$ giving $\left(e^{2} / \kappa L\right) /(2 \pi \gamma / L) \approx 0.16$. In the present numerical calculations, the parameter $\left(e^{2} / \kappa L\right) /(2 \pi \gamma / L)=0.16$ is used. We shall choose $\varepsilon_{c}=3 \gamma_{0}$ as in the case of single-wall tubes. This gives $\varepsilon_{c} /(2 \pi \gamma / L) \approx 10$ for typical nanotubes with diameter $2 R \sim 1.4 \mathrm{~nm}$. Calculations for slightly different values of these parameters give essentially the same results, although they are not shown here.

The exact value of $\kappa$ in carbon nanotubes is not known. Previous calculations for parallel polarization show that the exciton energy is almost independent of $\left(e^{2} / \kappa L\right) /(2 \pi \gamma / L)$ between 0.1 , corresponding to $\kappa \approx 4$, and 0.2 , corresponding to $\kappa \approx 2$, for both first and second gaps. ${ }^{6,19}$ For cross polarization, the exciton energy is more sensitive to the interaction parameter, suggesting $0.1<\left(e^{2} / \kappa L\right) /(2 \pi \gamma / L)<0.2{ }^{47}$ The two-photon energy is much more sensitive, concluding that $\left(e^{2} / \kappa L\right) /(2 \pi \gamma / L)$ should be around $0.15 \sim 0.16 .^{48}$

\section{EFFECTS OF INTERWALL SCREENING}

In this section and in the following, we shall consider a double-wall tube and confine ourselves to the case that at least one of the two tubes is semiconducting, in order to clarify roles of interwall screening. We shall denote the radius of the semiconducting tube by $R$ and assume $v_{R}=+1$. The other tube with radius $R^{\prime}$ can be either semiconducting $\left(v_{R^{\prime}}=+1\right)$ or metallic $\left(v_{R^{\prime}}=0\right)$. We vary $R^{\prime} / R$ for a wide range (both $R^{\prime} / R>1$ and $R^{\prime} / R<1$, corresponding to the tube with $R^{\prime}$ lying outside and inside, respectively).

The effective Coulomb interaction between two electrons on the cylinder surface with radius $R$ is explicitly calculated as

$$
V_{R R}(m, q)=\frac{V_{R R}^{0}(m, q)}{\tilde{\epsilon}_{R}(m, q)}
$$

with the effective dielectric function including interwall screening

$$
\tilde{\epsilon}_{R}(m, q)=\epsilon_{R}(m, q)+\frac{\left[\epsilon_{R^{\prime}}(m, q)-1\right] \tau_{m}(q)}{\epsilon_{R^{\prime}}(m, q)-\left[\epsilon_{R^{\prime}}(m, q)-1\right] \tau_{m}(q)},
$$

where

$$
\begin{gathered}
\epsilon_{R}(m, q)=\kappa+V_{R R}^{0}(m, q) \Pi_{R}(m, q), \\
\epsilon_{R^{\prime}}(m, q)=\kappa+V_{R^{\prime} R^{\prime}}^{0}(m, q) \Pi_{R^{\prime}}(m, q), \\
\tau_{m}(q)=\frac{I_{|m|}\left(|q| R_{\min }\right) K_{|m|}\left(|q| R_{\max }\right)}{K_{|m|}\left(|q| R_{\min }\right) I_{|m|}\left(|q| R_{\max }\right)} .
\end{gathered}
$$

The effective dielectric function $\tilde{\varepsilon}_{R^{\prime}}(m, q)$ and the screened potential $V_{R^{\prime} R^{\prime}}(m, q)$ for the other tube with $R^{\prime}$ can be obtained by exchanging $R$ and $R^{\prime}$.

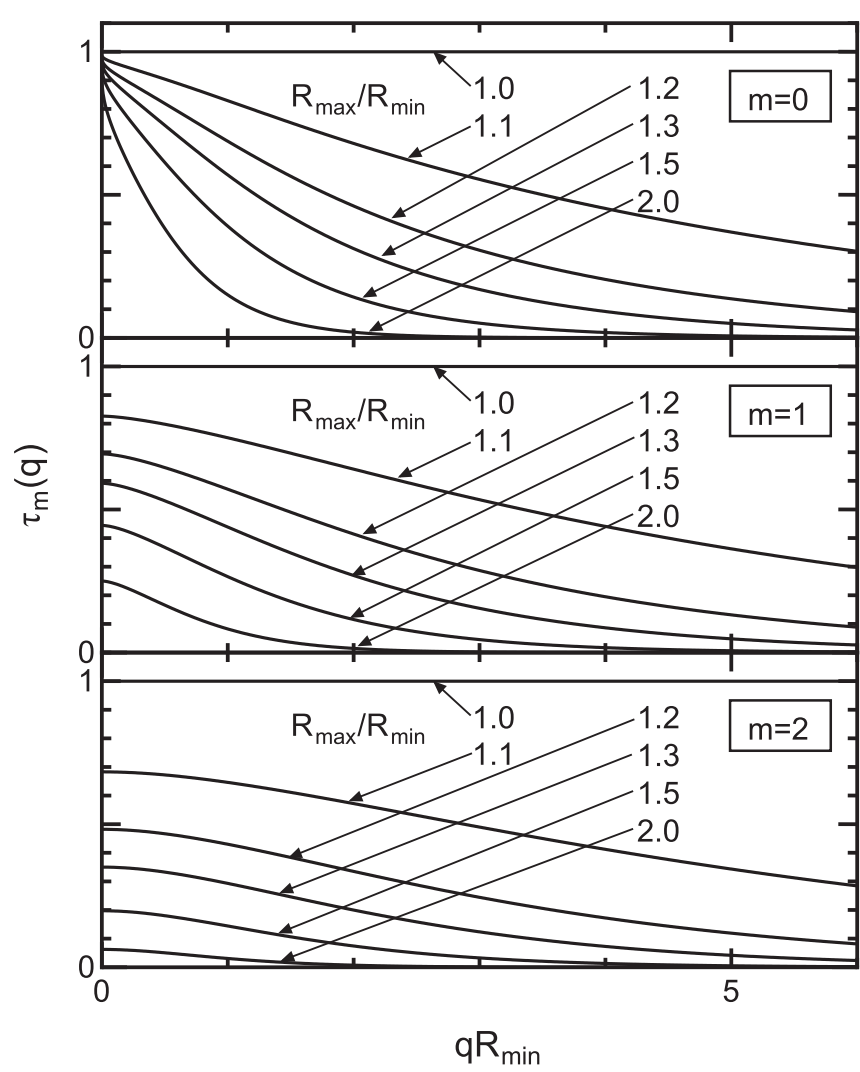

FIG. 3. Screening factor $\tau_{m}(q)$ as a function of $q R_{\min }$ for various values of $R_{\max } / R_{\min }$.

We can easily show $0 \leqslant \tau_{m}(q) \leqslant 1$. In the long-wavelength case $q R \ll 1$ and $q R^{\prime} \ll 1$, we have

$$
\tau_{m}(q) \simeq \begin{cases}1+\frac{\ln \left(R_{\max } / R_{\min }\right)}{\ln \left(e^{\gamma} q R_{\min } / 2\right)} & (m=0) \\ \left(\frac{R_{\min }}{R_{\max }}\right)^{2|m|} & (m \neq 0),\end{cases}
$$

with Euler's constant $\gamma=0.57721 \cdots$. In the shortwavelength case $q R \gg 1$ and $q R^{\prime} \gg 1$, we have

$$
\tau_{m}(q) \simeq \exp \left(-2 q\left|R-R^{\prime}\right|\right) .
$$

For $m=0, \tau_{m}(q)$ is singular and its derivative diverges at $q=0$ except in the special (unrealistic) case of $R^{\prime} / R=1$.

Figure 3 shows $\tau_{m}(q)$ for various values of $R_{\max } / R_{\min }$. First, we notice that the case of $R_{\max } / R_{\min }=1$ is exceptional, for which $\tau_{m}(q)=1$. In fact, a typical double-wall tube has $R_{\min } \sim 0.5 \mathrm{~nm}$ and $R_{\max } \sim 0.85 \mathrm{~nm}$ and, therefore, $R_{\max } / R_{\min } \gtrsim 1.5$. For such tubes, $\tau_{m}(q)$ generally decreases with $q$ and approaches 0 for sufficiently large $q$. This dependence becomes stronger with increasing $R_{\max } / R_{\min }$. For $m=0$ shown in the topmost panel, $\tau_{0}(q)$ suddenly decreases from 1 in the vicinity of $q=0$, corresponding to the singularity mentioned above. Because of the large $q$ dependence of $\tau_{m}(q)$, effects of constant $\kappa$ introduced in Eq. (17) cannot be represented by a background dielectric constant independent of $q$, as mentioned in the previous section. We note here that $\tau_{m}(q)$ is the same as $\tau_{m}\left(q R, q R^{\prime}\right)$ in Ref. 19, where environment effects are discussed.

Figure 4 shows some examples of the effective dielectric function $\tilde{\epsilon}_{R}(0, q)$ when the surrounding outer tube with radius 

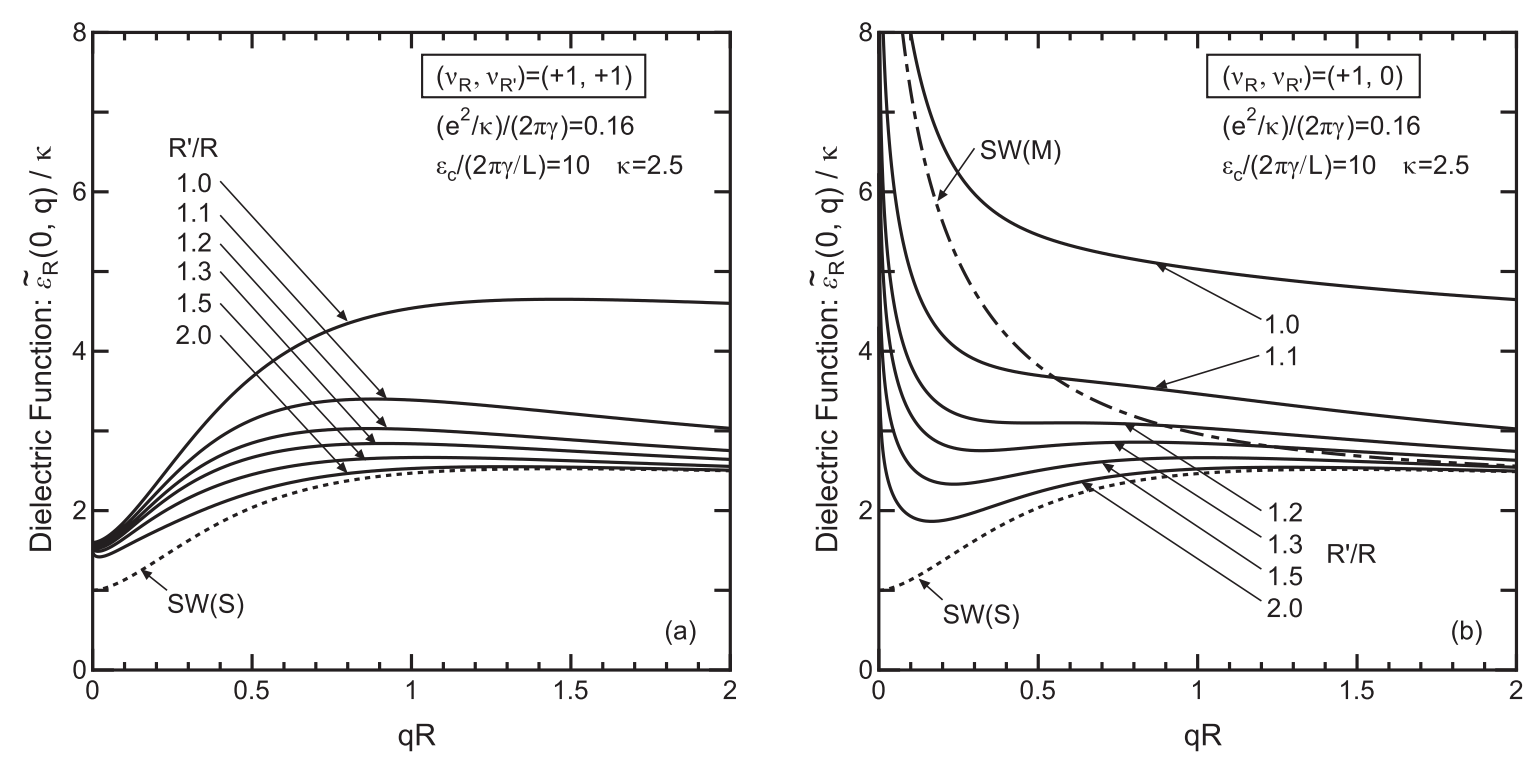

FIG. 4. The effective dielectric function $\tilde{\epsilon}_{R}(0, q)$ of a semiconducting nanotube with radius $R$. The outer tube is (a) semiconducting and (b) metallic. The dotted (dot-dashed) line denotes $\epsilon_{R}(0, q)$ of a semiconducting (metallic) single-wall tube.

$R^{\prime}$ is [Fig. 4(a)] semiconducting and [Fig. 4(b)] metallic. Only the results for $R^{\prime} \geqslant R$ are shown, but those for $R^{\prime}<R$ can immediately be obtained by exchanging $R$ and $R^{\prime}$.

In both Figs. 4(a) and 4(b), the dielectric function of the single-wall tube is shown by the dotted line. It increases from $\varepsilon_{R}(0,0)=\kappa$ gradually with $q$ and becomes almost independent of $q$ for $q R>1$, i.e., $\varepsilon_{R}(0, q)>\varepsilon_{R}(0,0)$ for $q R>1$. This $q$ dependence is responsible for the so-called antiscreening behavior in the single-wall nanotube. ${ }^{13}$ In fact, the screened potential $V_{R}(y)$ is reduced from $V_{R}^{0}(y) / \kappa$ in the short-distance region $(|y| \lesssim R)$ but is enhanced over $V_{R}^{0}(y) / \kappa$ in the longdistance region $(|y| \gtrsim R)$.

In double-wall tubes consisting of two semiconducting tubes, except in the unrealistic case of $R^{\prime} / R=1$, the amount of the increase of $\tilde{\varepsilon}_{R}(0, q)$ with increasing $q$ is generally reduced as shown in Fig. 4(a), causing the reduction of the antiscreening behavior. For tubes with $R_{\max } / R_{\min }>1.5$, the effective dielectric function becomes closer to a constant independent of $q$ without the antiscreening. ${ }^{49}$ When the other tube is metallic, on the other hand, $\tilde{\varepsilon}_{R}(0, q) \rightarrow \infty$ for $q \rightarrow 0$ because $\Pi_{R^{\prime}}(0, q) \rightarrow 2 g_{s} /(\pi \gamma)$, and, therefore, the effective potential is screened out in the long-distance region, completely destroying the antiscreening behavior.

When the other tube is semiconducting, we have $\tilde{\varepsilon}_{R}(0,0)=$ $2 \kappa-1(=4$ for $\kappa=2.5)$ at $q=0$ because $\tau_{0}(0)=1$. The singularity in $\tau_{0}(q)$ at $q=0$, shown in Eq. (37) and in Fig. 3, gives rise to a sharp decrease of $\tilde{\varepsilon}_{R}(0, q)$ from $2 \kappa-1$ with $q$ and causes the appearance of a minimum in the vicinity of $q=0$ as shown in Fig. 4(a). The effective Coulomb potential for excitons in the long-distance region is essentially determined by the minimum value of $\tilde{\varepsilon}_{R}(0, q)$ rather than $\tilde{\varepsilon}_{R}(0,0)=$ $2 \kappa-1$.

Figure 5(a) shows calculated excitation energies as a function of the radius ratio $R^{\prime} / R$ when the other tube with radius $R^{\prime}$ is semiconducting. These energies are shown for the first $(n=0)$ and second $(n=1)$ gaps. The result in Fig. 5(a) clearly shows that, in the presence of inner or outer tube, exciton energy levels and band gap are redshifted from those in a single-wall tube with the same diameter. This redshift is mainly due to that of the band gap because the exciton binding energies are reduced by interwall screening, as will be shown below. The energy level of the ground exciton approaches that of the single-wall tube rapidly away from $R^{\prime} / R=1$, resulting from a large cancellation of the band gap reduction and the decrease of the binding energy by screening. On the other hand, excited excitons undergo large energy shifts accompanying the band gap shift but remain as bound states for any value of $R^{\prime} / R$.

Figure 5(b) shows calculated excitation energies when the other tube is metallic. The band gap reduction from the singlewall tube is much larger. However, the ground exciton still exists with a significant binding energy except in the vicinity of $R^{\prime} / R=1$ and its energy level is nearly the same as that in Fig. 5(a). The most striking feature of the metallic screening is that excited exciton states disappear in the region $0.5 \lesssim$ $R^{\prime} / R \lesssim 2$ for the first gap and in the region $0.7 \lesssim R^{\prime} / R \lesssim 1.5$ for the second gap. This becomes more apparent in the exciton binding energy as shown below.

Figure 6(a) shows exciton binding energies of the first gap in the semiconducting case corresponding to Fig. 5(a). It is interesting to notice that the binding energy most strongly depends on radius ratio $R^{\prime} / R$ for the ground exciton and the dependence becomes weaker for the excited excitons. This different behavior can be understood by Fig. 4(a), which shows that the screening in the long-distance region, corresponding to $q R \ll 1$ and contributing most to excited states, is weakly dependent on $R^{\prime} / R$, while that in the short-distance region corresponding to $q R \gtrsim 1$, contributing most to the ground exciton state, is very sensitive to the slight increase in $R^{\prime} / R$. In the real space, this corresponds to the fact that the shortrange part of the effective electron-hole Coulomb potential $(|y| \lesssim L$ with $y$ being the relative coordinate for an electronhole pair and $L=2 \pi R$ ) is more sensitive to $R^{\prime} / R$, but the long-range part $(|y| \gtrsim L)$ is less sensitive, although not shown here. 

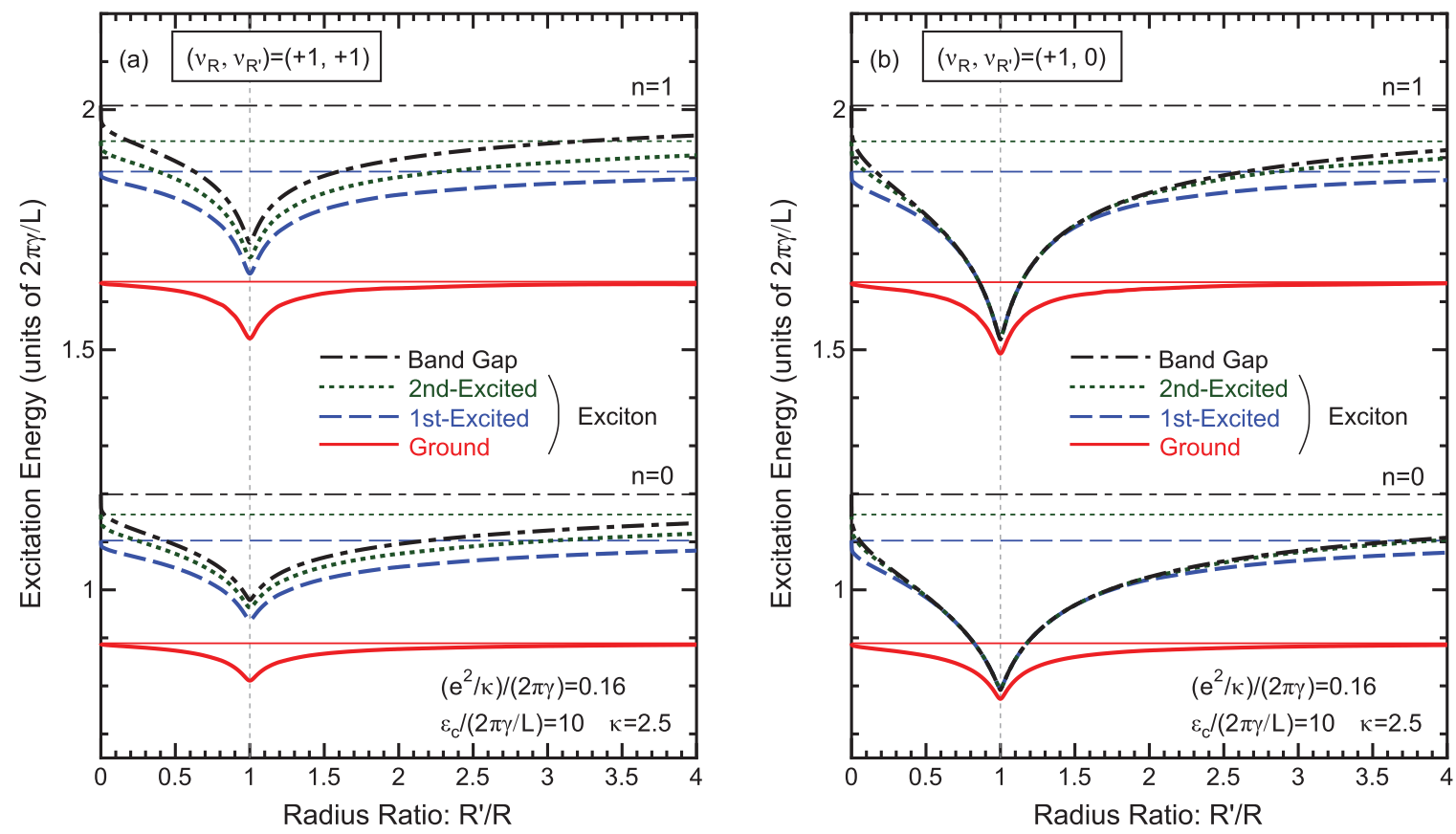

FIG. 5. (Color online) Calculated excitation energies of a semiconducting nanotube with radius $R$ as a function of $R^{\prime} / R$. The other tube with $R^{\prime}$ is (a) semiconducting and (b) metallic. The thin horizontal lines denote the results in the single-wall tube.

Figure 6(b) shows exciton binding energies of the first gap in the metallic case corresponding to Fig. 5(b). We can see the intense reduction of the binding energies and the vanishing of excited exciton states in the region $0.5 \lesssim R^{\prime} / R \lesssim 2$. In other regions, the first- and second-excited exciton states appear but higher excited exciton states are absent. The features of exciton binding energies in the second gap are qualitatively the same as in the first gap for both semiconducting and metallic cases.

Figure 7 shows the oscillator strengths of the ground and second-excited excitons of the first gap. The first-excited exciton is optically inactive because of its parity but can be observed in two-photon processes. ${ }^{10,11,48,50}$ The reduction of the oscillator strength is not so appreciable for the ground exciton for both semiconducting and metallic outer tube except in the vicinity of $R^{\prime} / R=1$. The metallic screening due to the outer tube drastically modifies the excited exciton state, resulting in the disappearance for $0.5 \lesssim R^{\prime} / R \lesssim 2$ as seen in Fig. 6(b). This well corresponds to Fig. 5(b).

Figure 8 shows some examples of the dynamical conductivity describing the absorption spectra of the semiconducting tube with radius $R$ surrounded by (a) semiconducting and (b) metallic outer tube for typical cases of $R^{\prime} / R=1.5$. In the case of a semiconducting outer tube [Fig. 8(a)], the absorption peak of the ground exciton, which is strongest, is slightly
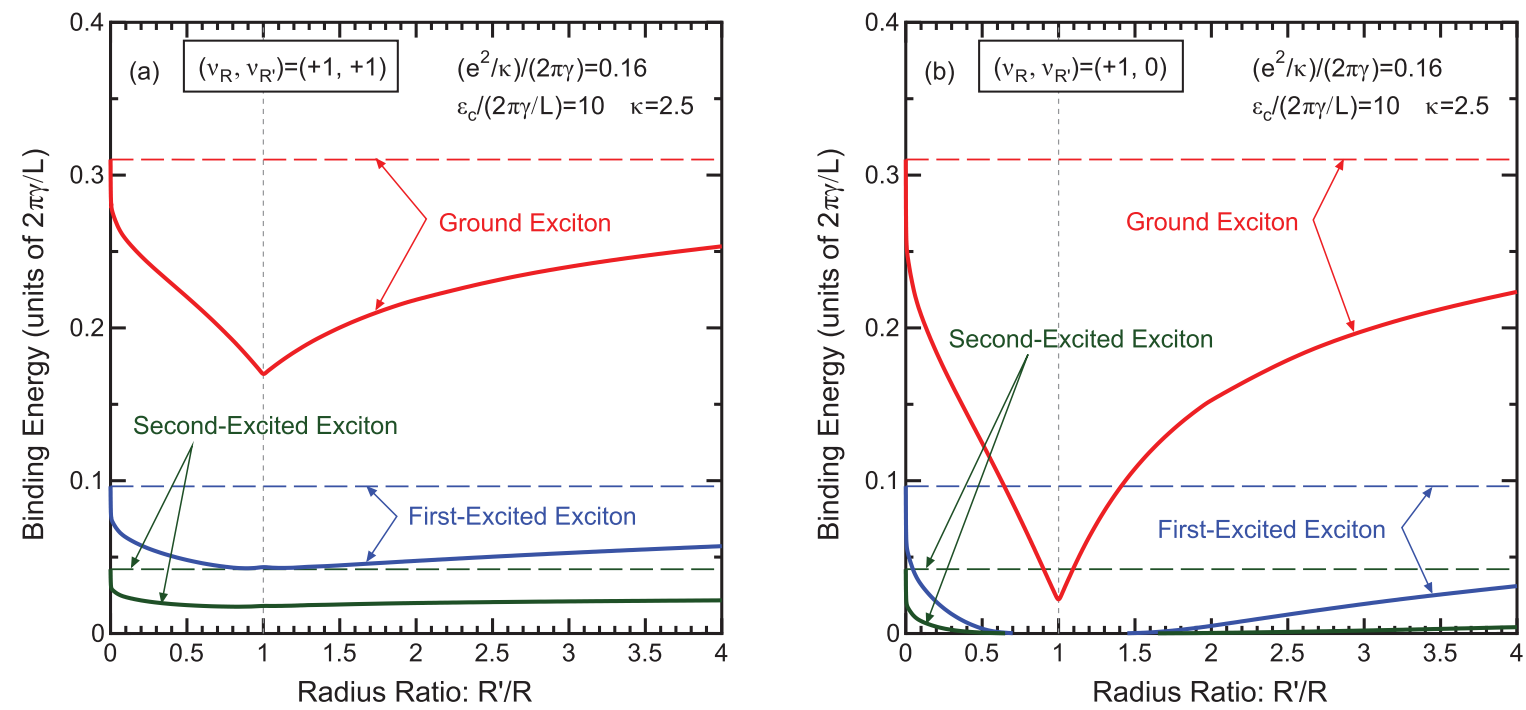

FIG. 6. (Color online) Exciton binding energies of the first gap corresponding to Fig. 5. The dashed lines represent those in the single-wall tube. 

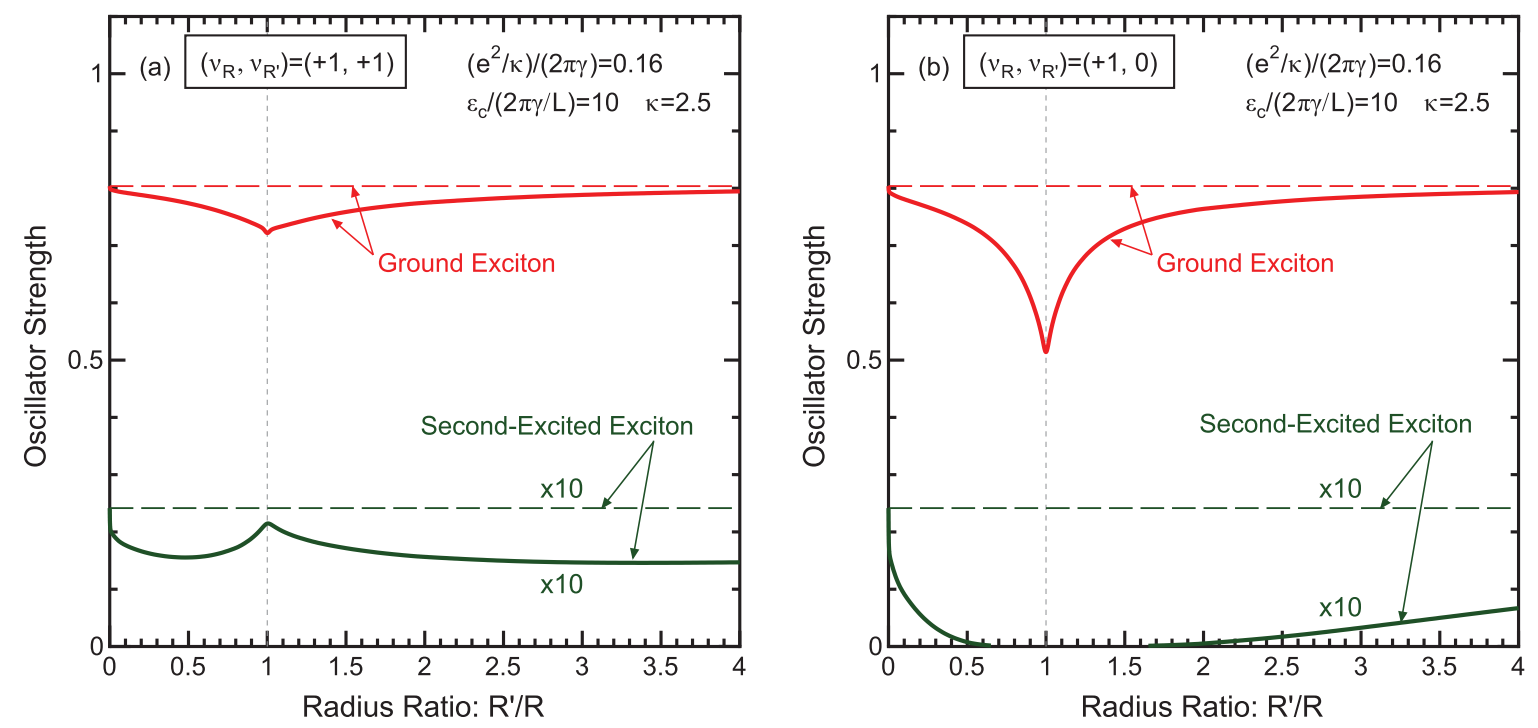

FIG. 7. (Color online) Oscillator strength of the ground and second excited excitons of the first gap corresponding to Fig. 5. The dashed lines represent the results in the single-wall tube.

redshifted in comparison with that of a single-wall tube. The redshift of the peak associated with the second excited exciton is much more prominent and the position becomes closer to the interband continuum.

In the case that the outer tube is metallic [Fig. 8(b)], the spectrum consists of a single dominant peak associated with the ground exciton and weak interband continuum, corresponding to the disappearance of excited exciton states as discussed above. The redshift of the exciton peak is slightly larger than that in the case of a semiconducting outer tube shown in Fig. 8(a), but the difference is not so appreciable. The reduction of the band gap is most noticeable.

The behavior of the exciton binding may be most straightforwardly seen in an effective real-space potential. The Coulomb potential between two ring charges in the tube with

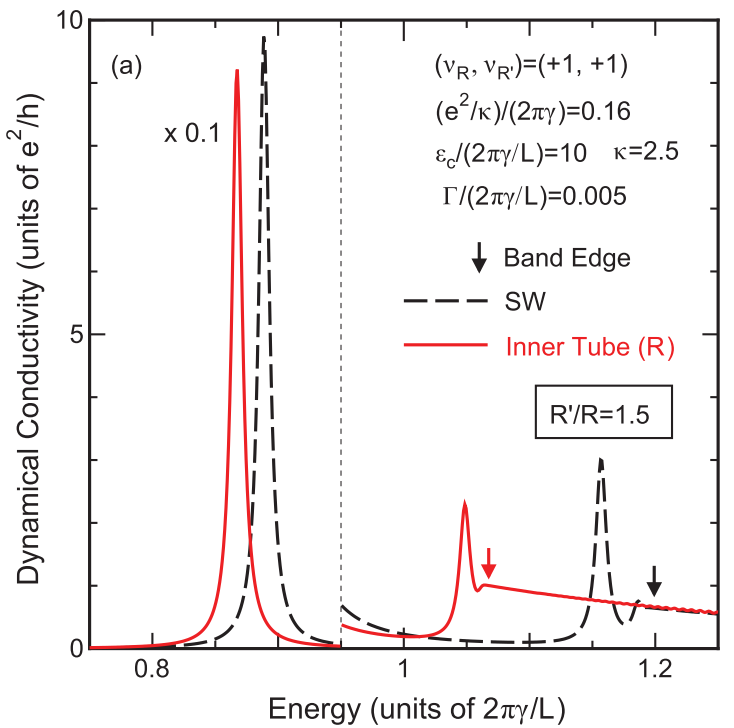

radius $R$ and the corresponding exciton wave function are given by the Fourier transform:

$$
\begin{gathered}
V_{R R}(y)=\int \frac{d q}{2 \pi} V_{R R}(0, q) \exp (i q y), \\
\psi_{R}^{u}(y)=\sqrt{\frac{A}{L}} \int \frac{d k}{2 \pi} \psi_{R, 0}^{u}(k) \exp (i k y),
\end{gathered}
$$

with $L=2 \pi R$. Figure 9 shows the screened potential for $R^{\prime} / R=1.5$ together with the exciton bound levels and the exciton wave functions. For reference, the screened potential in a semiconducting single-wall tube (the dotted line) is shown in both Figs. 9(a) and 9(b). The corresponding exciton bound levels are represented by the horizontal lines, whose length

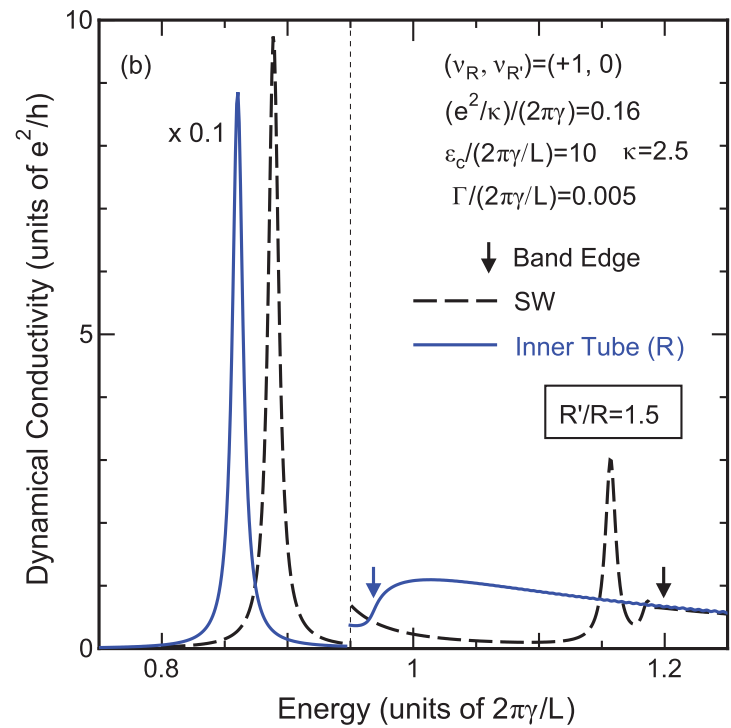

FIG. 8. (Color online) Calculated dynamical conductivity of a semiconducting nanotube with radius $R$ near the ground exciton of the first gap in the presence of a (a) semiconducting and (b) metallic outer tube with radius $R^{\prime}$ for $R^{\prime} / R=1.5$. The dashed lines denote the conductivity in the single-wall tube. The arrows denote the bottom of the interband continuum. 


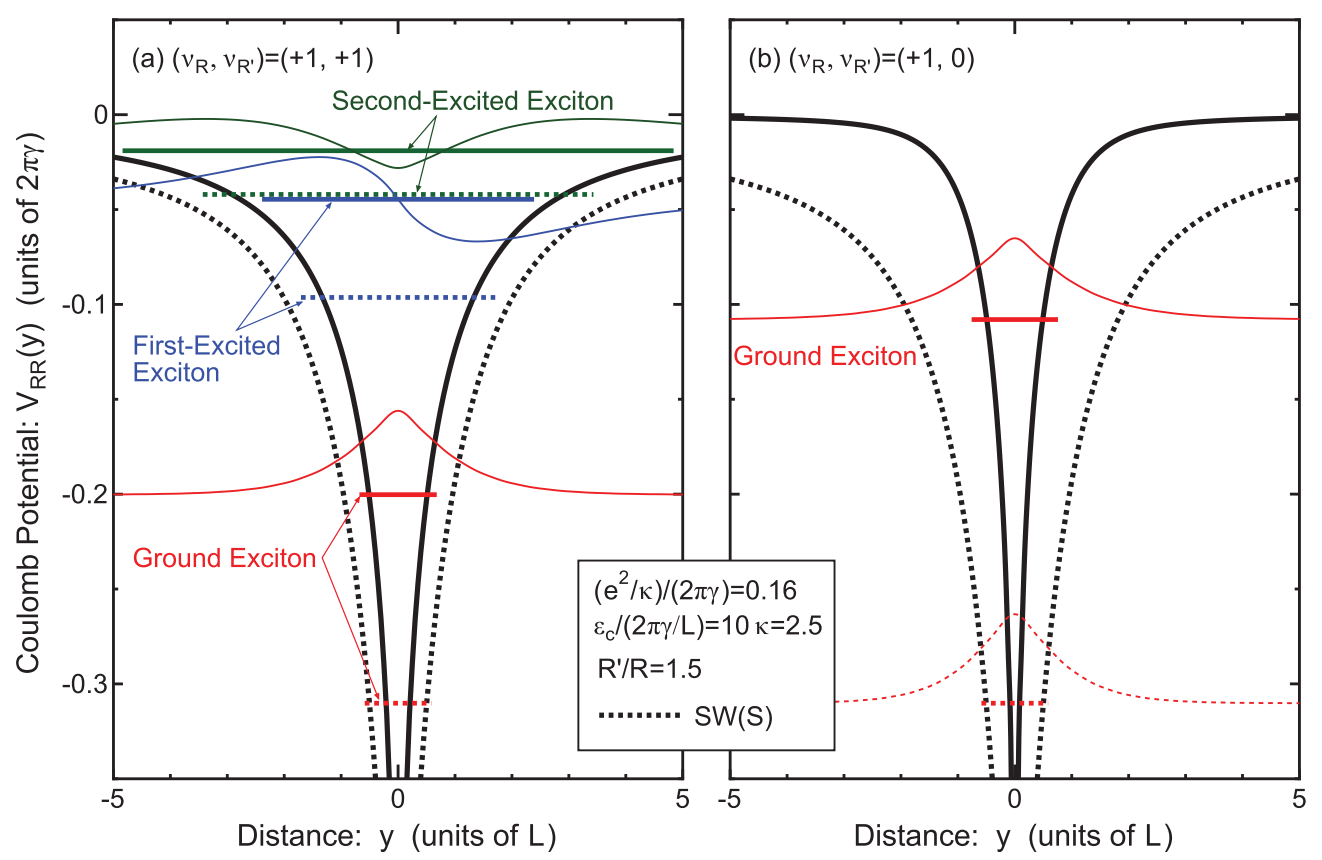

FIG. 9. (Color online) The screened Coulomb potential for an electron-hole pair in a semiconducting nanotube with radius $R$ surrounded by an outer tube with radius $R^{\prime}$ for $R^{\prime} / R=1.5$, together with the exciton wave functions. (a) The outer tube is semiconducting and (b) metallic. The horizontal lines stand for exciton bound levels and their length indicates the spatial extent $2 \sqrt{\left\langle y^{2}\right\rangle}$. In (b) the results for the ground exciton in single-wall tube are shown by the dotted lines.

represents the spatial extent $2 \sqrt{\left\langle y^{2}\right\rangle}$, where the average is taken using $\psi_{R}^{u}(y)$.

In the presence of a semiconducting or metallic outer tube, the potential is reduced from that of the single-wall tube in the whole region. For a semiconducting outer tube, in particular, the tendency of the antiscreening is still present, although being not so clear in Fig. 9(a). As a result, excited exciton states (first and second) are not removed by the interwall screening, although the binding energy is reduced and the wave function becomes more extended. In fact, the spatial extent in the double-wall tube is $2 \sqrt{\left\langle y^{2}\right\rangle} / L \simeq 4.8$ for the first-excited exciton and 9.7 for the second-excited exciton, in contrast to 3.4 and 6.9 , respectively, in the single-wall tube. For a metallic outer tube, on the other hand, the potential in the long-distance region is completely screened out, destroying all excited exciton states.

In both Figs. 9(a) and 9(b), the ground exciton remains as a bound state and its spatial extent does not change so much from the single-wall case and remains about the same as the tube circumference, i.e., $\sim L$. In fact, the spatial extent is $2 \sqrt{\left\langle y^{2}\right\rangle} / L \simeq 1.2$ in the single-wall tube and becomes 1.4 and 1.5 for a semiconducting and metallic outer tube, respectively, in the double-wall tube. The increase of $2 \sqrt{\left\langle y^{2}\right\rangle}$ is much smaller than for excited exciton states.

This behavior of the ground-exciton wave function is closely related to the infinite binding energy for potential $\propto-1 /|y|$ in a real one-dimensional system due to a fall of a particle into $y=0 .^{51,52}$ In carbon nanotubes with circumference $L$, the potential is effectively cut off around $|y| \sim L$ and, therefore, the exciton binding energy becomes finite. As a result, the spatial extent of the wave function is almost always $\sim L$ independent of screening. The present result shows that the interwall screening does not modify this feature of the one-dimensionality of excitons even in double-wall tubes.

\section{DIAMETER DEPENDENCE}

In double-wall tubes, the interwall distance is usually about the same as that of inter layer distance in bulk graphite. ${ }^{53,54}$ Thus, it is straightforward to calculate excitation energies and optical absorption spectra for varying diameter in double-wall tubes if we set $R^{\prime}-R=0.34 \mathrm{~nm}$ or $R-R^{\prime}=0.34 \mathrm{~nm}$. Figure 10 shows the ground exciton energies and the bottoms of the interband continua of double-wall nanotubes as a function of the tube diameter. A semiconducting tube with radius $R$ is surrounded by a semiconducting tube with radius $R^{\prime}\left(R^{\prime}>\right.$ $R$ ) in (a), it is surrounded by a metallic tube with radius $R^{\prime}$ in (b), and it surrounds a metallic inner tube with $R^{\prime}\left(R^{\prime}<R\right)$. The lower horizontal axis shows the diameter of the semiconducting tube and the upper horizontal axis shows the diameter of the outer semiconducting tube in (a), the diameter ratio $R^{\prime} / R$ in (b), and the diameter of inner metallic tube in (c).

In Fig. 10(a) for double-wall tubes consisting of two semiconducting tubes, excitation energies associated with the first and second gaps are shown. In Figs. 10(b) and 10(c), the exciton energy and the bottom of interband continuum for the second gap consisting of $n= \pm 1$ are shown in metallic nanotubes as well as the excitation energies of the semiconducting tube. In metallic nanotubes, optical transition is forbidden between the conduction and valence bands with $n=0$ having a linear dispersion but becomes allowed between the bands with $n= \pm 1$, for which an exciton bound state is formed even in the presence of metallic screening by electrons in the linear bands. ${ }^{47,55}$ We notice that the shift due to inter wall screening is slightly larger in the case of 


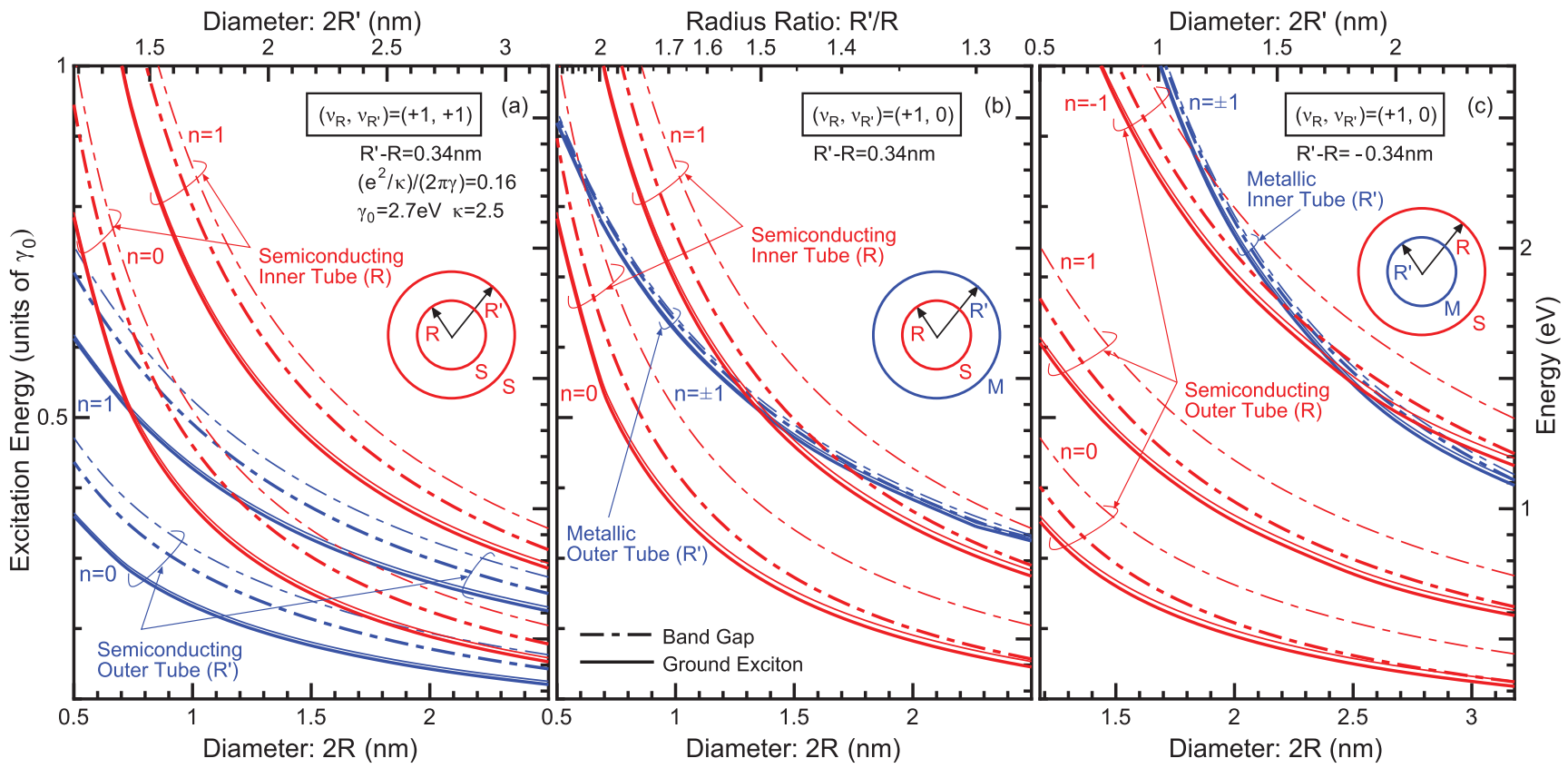

FIG. 10. (Color online) The ground exciton energies (solid lines) and the bottoms of the interband continua (dot-dashed lines) for the fixed interwall distance at $R^{\prime}-R= \pm 0.34 \mathrm{~nm}$ as a function of the tube diameter, (a) consisting of both semiconducting tubes, (b) a semiconducting tube surrounded by a metallic outer tube, and (c) a semiconducting tube surrounding a metallic inner tube. The thick lines represent the results in double-wall tubes and the thin lines those in single-wall tubes.

metallic interwall screening than in the case of semiconducting interwall screening, although its absolute value is very small in the energy scale of the figure.

In Fig. 10(a) where both tubes are semiconducting, the exciton of the second gap in the outer tube crosses the exciton of the first gap in the inner tube around $2 R \simeq 0.75 \mathrm{~nm}$ ( $R^{\prime} / R \simeq 1.8$ ). This crossing point is about the same without interwall screening, which is in agreement with recent experiments. ${ }^{15}$ In single-wall tubes, this crossing is determined by $E_{11}(R)=E_{22}\left(R^{\prime}\right)$. We obtain $R^{\prime} / R \simeq 1.8$ by noting

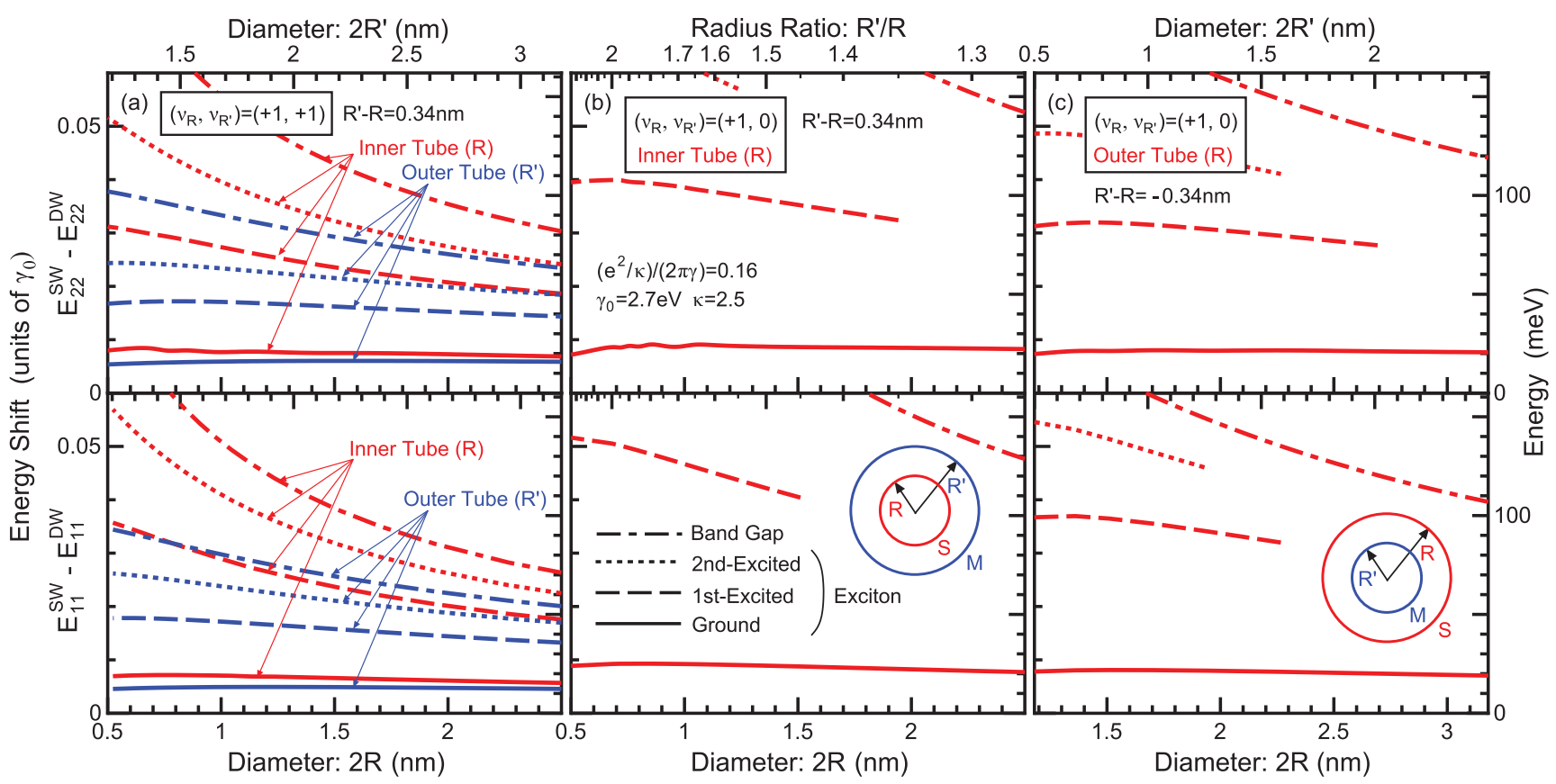

FIG. 11. (Color online) The shift of excitation energies due to the presence of an outer or inner tube for the fixed interwall distance at $R^{\prime}-R= \pm 0.34 \mathrm{~nm}$ as a function of the tube diameter, (a) consisting of both semiconducting tubes, (b) a semiconducting tube surrounded by a metallic outer tube, and (c) a semiconducting tube surrounding a metallic inner tube. The lower and upper panels show the energy shifts for the first and second gaps, respectively. 


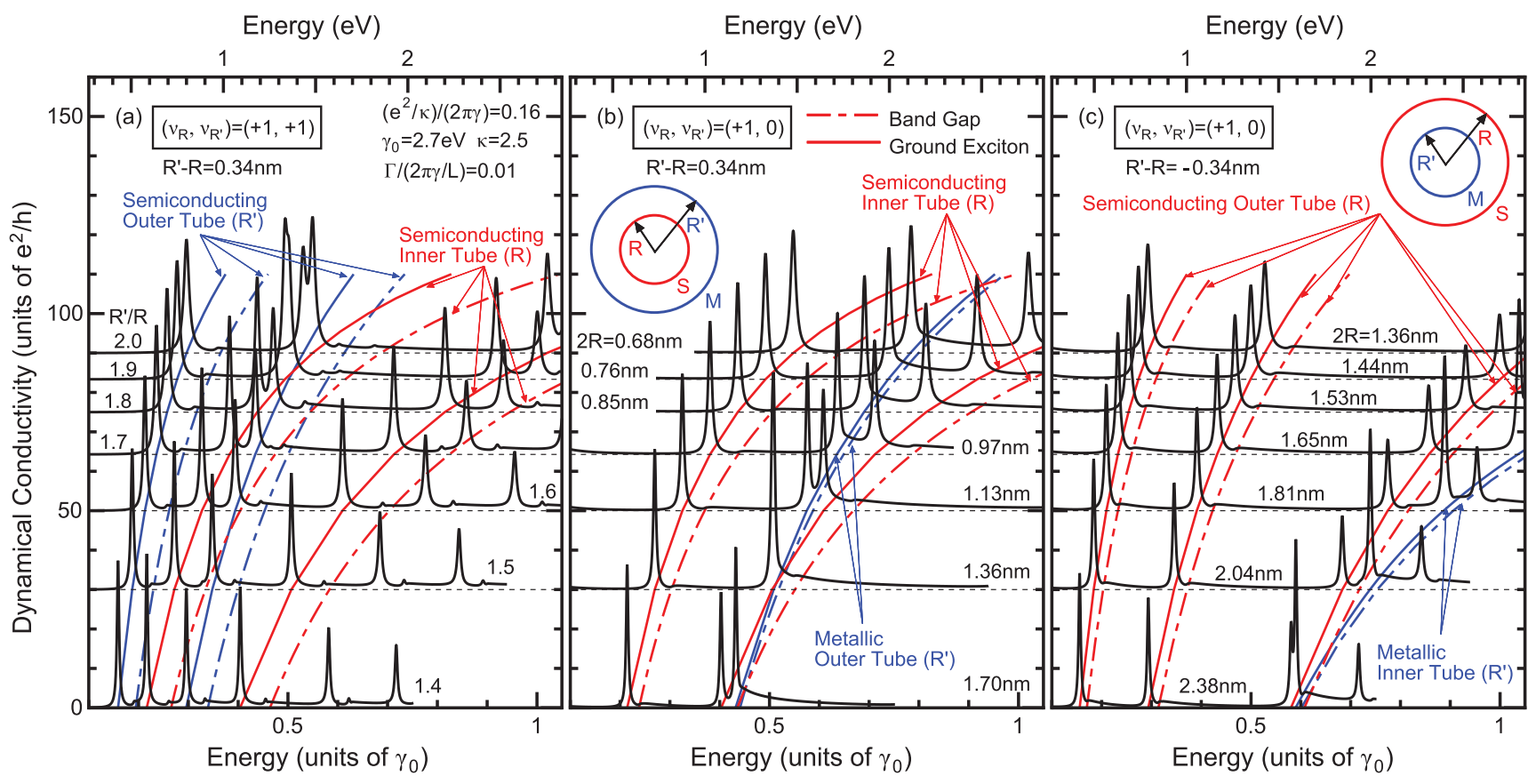

FIG. 12. (Color online) Calculated dynamical conductivity $\sigma(\omega)$ of a double-wall nanotube with the fixed interwall distance at $R^{\prime}-R=$ $\pm 0.34 \mathrm{~nm}$, consisting of (a) two semiconducting tubes, (b) a semiconducting inner tube and a metallic outer tube, and (c) a metallic inner tube and a semiconducting outer tube. The tube diameter is varied from $2 R=0.68 \mathrm{~nm}$ to $1.7 \mathrm{~nm}$ in (a) and (b) and $2 R=1.36 \mathrm{~nm}$ to $2.38 \mathrm{~nm}$ in (c). The solid and dash-dotted lines represent the ground exciton energies and the bottoms of the interband continua, respectively.

$E_{22}(R) \approx 1.8 \times E_{11}(R)$ and $E_{11}(R) \propto E_{22}(R) \propto R^{-1} \cdot 6,9$ In Fig. 10(b), the exciton in the metallic outer tube crosses the exciton of the second gap in the semiconducting inner tube in the region $1.3 \lesssim 2 R \lesssim 1.4 \mathrm{~nm}$. Because the redshift due to interwall screening is larger, this crossing occurs in the double-wall tube at a diameter that slightly differs from that in single-wall nanotubes.

Figure 11 shows the shift of excitation energies due to the presence of an outer or inner tube. The shift of the ground exciton is almost independent of the diameter and remains very small, while those of exciton excited states and band gaps are much larger and decrease with the diameter. For a double-wall tube consisting of two semiconducting tubes, the shift of the inner tube lies between $\sim 15$ and $\sim 20 \mathrm{meV}$ and that of the outer tube is slightly smaller. When the semiconducting tube is surrounded by or surrounding a metallic tube, the shift of the ground exciton lies between $\sim 20$ and $\sim 30 \mathrm{meV}$ and excited excitons are present only in very narrow tubes and disappear beyond a critical value of the diameter.

With increasing $R$, the ratio $R^{\prime} / R$ decreases and therefore the screening effect becomes relatively larger as shown in Figs. 5 and 6. However, the energy scale itself decreases with $R$ because excitation energies are all scaled roughly by $2 \pi / L \propto$ $R^{-1}$. These two effects tend to cancel each other for the ground exciton, giving rise to the shifts almost independent of the tube diameter. For excited excitons and band gaps, the dependence on $R^{\prime} / R$ is much weaker and, therefore, their shifts due to interwall screening decrease with the diameter, because of the dominant $2 \pi / L \propto R^{-1}$ scaling of their energies.

Figure 12 shows some examples of calculated absorption spectra, $\operatorname{Re} \sigma(\omega)$, in double-wall tubes consisting of (a) two semiconducting tubes, (b) a semiconducting inner tube and a metallic outer tube, and (c) a metallic inner tube and a semiconducting outer tube, corresponding to Fig. 11. Both inner and outer tubes almost equally contribute to the absorption, being dominated by excitons. This feature is essentially independent of whether tubes are metallic or semiconducting.

\section{DISCUSSION AND SUMMARY}

Photoluminescence experiments were performed in doublewall-rich samples showing shifts from the single-wall tubes as small as a few $\mathrm{meV}^{14,15}$ or vanishingly small. ${ }^{23}$ The present calculation also gives a small shift, which is almost invisible in Fig. 10. As shown in Fig. 11, however, this small shift is almost one order of magnitude larger than the experiments. Origins of this discrepancy are not known and should be clarified in future.

Experimentally, photoluminescence intensity seems to be modified by interwall interactions in double-wall tubes. In fact, observed increase was ascribed to exciton transfer from $E_{22}$ of outer tubes to $E_{11}$ of inner tubes ${ }^{15}$ and observed suppression was considered to be due to strong quenching by metallic outer tubes. ${ }^{25}$ The calculated oscillator strength in Fig. 7 shows a small decrease in absorption intensity due to interwall screening but is certainly not directly related to the photoluminescence intensity.

Previous calculations of interwall conductance show that interwall hopping is strongly enhanced in the presence of disorder due to destruction of the cancellation of the interwall coupling at different carbon sites, ${ }^{56}$ suggesting that strength of exciton transfer is sensitive to disorder. The photoluminescence intensity is highly likely to depend on relative strength of interwall transfer and radiative recombination of excitons. 
The small shift of the ground exciton energy in double-wall tubes is a result of the cancellation of the reduction in the band gap due to the interwall screening and the reduction in the exciton binding energy. This cancellation is exact, if we consider only the "long-range part" of the Coulomb potential $(q R \lesssim 1)$ in calculating the self-energy and the exciton binding energy. The small shift appears due to "short-range part" of the Coulomb interaction $(q R \gtrsim 1)$ and mixings between different bands. The interwall screening also modifies these residual interactions and, therefore, gives rise to the small shift from the single-wall case, very roughly about $10-15 \mathrm{meV}$ for double-wall tubes consisting of two semiconducting tubes, as discussed above.

In graphene, the interband conductivity is known to be given by $(\pi / 2)\left(e^{2} / h\right)$ independent of frequency, ${ }^{57}$ giving rise to absorption of $\sim 2.3 \%$ per sheet as experimentally observed. ${ }^{58,59}$ Figure 12 shows that the peak conductivity in nanotubes can be as large as $\sim 40\left(e^{2} / h\right)$, although the peak height itself is sensitive to the broadening and, therefore, varies depending of quality of nanotubes. This is more than one order of magnitude larger than that of graphene and causes strong absorption of incident light. In the case that the exciton levels in different tubes happen to overlap with each other, Eq. (31) might be modified and an adequate treatment is likely to be needed for a self-consistent determination of the electric field at different tubes. This problem is out of the scope of this paper and left for future study.
In summary, we have studied effects of interwall screening on excitons and optical spectra in double-wall carbon nanotubes in the static screened Hartree-Fock approximation within a $\boldsymbol{k} \cdot \boldsymbol{p}$ scheme. The intrawall electron-hole interaction is largely suppressed by interwall screening effects. The suppression is sensitive to semiconducting or metallic screening and also to the effective interwall distance between the inner and outer tubes. This suppression reduces the exciton binding energy as well as the band gap energy. These two effects almost cancel with each other, causing a small redshift for the energy of the ground exciton from that in single-wall tubes with the same diameter. Further, this shift of the ground exciton has little dependence on the tube diameter. Excited exciton states are strongly affected by the interwall screening and are strongly suppressed (and even disappear) when the other tube is metallic.

\section{ACKNOWLEDGMENT}

This work was supported in part by a Grant-in-Aid for Scientific Research on Innovative Area "Optical Science of Dynamically Correlated Electrons" (20104009), by a Grantin-Aid for Scientific Research on Priority Area "Carbon Nanotube Nanoelectronics" (19054004), and by Grants-in-Aid for Scientific Research (C) $(20540307,22540326)$ from the Ministry of Education, Culture, Sports, Science and Technology, Japan. *tomio@eng.hokudai.ac.jp

${ }^{1}$ R. Saito, G. Dresselhaus, and M. S. Dresselhaus, Physical Properties of Carbon Nanotubes (Imperial College Press, London, 1998). ${ }^{2}$ T. Ando, J. Phys. Soc. Jpn. 74, 777 (2005).

${ }^{3}$ A. H. Castro Neto, F. Guinea, N. M. Peres, K. S. Novoselov, and A. K. Geim, Rev. Mod. Phys. 81, 109 (2009).

${ }^{4}$ J.-C. Charlier, X. Blase, and S. Roche, Rev. Mod. Phys. 79, 677 (2007).

${ }^{5}$ T. Ando, J. Phys. Soc. Jpn. 66, 1066 (1997).

${ }^{6}$ T. Ando, J. Phys. Soc. Jpn. 73, 3351 (2004).

${ }^{7}$ C. D. Spataru, S. Ismail-Beigi, L. X. Benedict, and S. G. Louie, Appl. Phys. A 78, 1129 (2004).

${ }^{8}$ V. Perebeinos, J. Tersoff, and Ph. Avouris, Phys. Rev. Lett. 92, 257402 (2004).

${ }^{9}$ M. Ichida, S. Mizuno, Y. Tani, Y. Saito, and A. Nakamura, J. Phys. Soc. Jpn. 68, 3131 (1999).

${ }^{10}$ F. Wang, G. Dukovic, L. E. Brus, and T. F. Heinz, Science 308, 838 (2005).

${ }^{11}$ J. Maultzsch, R. Pomraenke, S. Reich, E. Chang, D. Prezzi, A. Ruini, E. Molinari, M. S. Strano, C. Thomsen, and C. Lienau, Phys. Rev. B 72, 241402 (2005).

${ }^{12}$ Y. Kanemitsu, PhysChemChemPhys 13, 14879 (2011).

${ }^{13}$ J. Deslippe, M. Dipoppa, D. Prendergast, M. V. O. Moutinho, R. B. Capaz, and S. G. Louie, Nano. Lett. 9, 1330 (2009).

${ }^{14}$ H. Hirori, K. Matsuda, and Y. Kanemitsu, Phys. Rev. B 78, 113409 (2008).

${ }^{15}$ D. Shimamoto, H. Muramatsu, T. Hayashi, Y. A. Kim, M. Endo, J. S. Park, R. Saito, M. Terrones, and M. S. Dresselhaus, Appl. Phys. Lett. 94, 083106 (2009).
${ }^{16}$ Y. Ohno, S. Iwasaki, Y. Murakami, S. Kishimoto, S. Maruyama, and T. Mizutani, Phys. Rev. B 73, 235427 (2006).

${ }^{17}$ Y. Ohno, S. Iwasaki, Y. Murakami, S. Kishimoto, S. Maruyama, and T. Mizutani, Phys. Stat. Sol. B 244, 4002 (2007).

${ }^{18}$ Y. Miyauchi, R. Saito, K. Sato, Y. Ohno, S. Iwasaki, T. Mizutani, J. Jiang, and S. Maruyama, Chem. Phys. Lett. 442, 394 (2007).

${ }^{19}$ T. Ando, J. Phys. Soc. Jpn. 79, 024706 (2010).

${ }^{20}$ C. Shen, A. H. Brozena, and Y. H. Wang, Nanoscale 3, 503 (2011).

${ }^{21}$ M. Endo, H. Muramatsu, T. Hayashi, Y. A. Kim, M. Terrones, and M. S. Dresselhaus, Nature 433, 476 (2005).

${ }^{22}$ T. Okazaki, S. Bandow, G. Tamura, Y. Fujita, K. Iakoubovskii, S. Kazaoui, N. Minami, T. Saito, K. Suenaga, and S. Iijima, Phys. Rev. B 74, 153404 (2006).

${ }^{23}$ H. Muramatsu, T. Hayashi, Y. A. Kim, D. Shimamoto, M. Endo, V. Meunier, B. G. Sumpter, M. Terrones, and M. S. Dresselhaus, Small 5, 2678 (2009).

${ }^{24}$ A. A. Green and M. C. Hersam, Nat. Nano. 4, 64 (2009).

${ }^{25}$ D. A. Tsyboulski, Y. Hou, N. Fakhri, S. Ghosh, R. Zhang, S. M. Bachilo, M. Pasquali, L. Chen, J. Liu, and R. B. Weisman, Nano. Lett. 9, 3282 (2009).

${ }^{26}$ F. Villalpando-Paez, L. G. Moura, C. Fantini, H. Muramatsu, T. Hayashi, Y. A. Kim, M. Endo, M. Terrones, M. A. Pimenta, and M. S. Dresselhaus, Phys. Rev. B 82, 155416 (2010).

${ }^{27}$ J. Alfonsi and M. Meneghetti, New J. Phys. 11, 043002 (2009).

${ }^{28}$ R. Moradiana and A. Fathaliana, Solid State Commun. 149, 491 (2009).

${ }^{29}$ J. W. McClure, Phys. Rev. 104, 666 (1956).

${ }^{30}$ J. C. Slonczewski and P. R. Weiss, Phys. Rev. 109, 272 (1958).

${ }^{31}$ D. P. DiVincenzo and E. J. Mele, Phys. Rev. B 29, 1685 (1984). 
${ }^{32}$ G. W. Semenoff, Phys. Rev. Lett. 53, 2449 (1984).

${ }^{33}$ H. Ajiki and T. Ando, J. Phys. Soc. Jpn. 62, 1255 (1993).

${ }^{34}$ C. L. Kane and E. J. Mele, Phys. Rev. Lett. 78, 1932 (1997).

${ }^{35}$ M. Kociak, K. Suenaga, K. Hirahara, Y. Saito, T. Nakahira, and S. Iijima, Phys. Rev. Lett. 89, 155501 (2002).

${ }^{36}$ J. M. Zuo, I. Vartanyants, M. Gao, R. Zhang, and L. A. Nagahara, Science 300, 1419 (2003).

${ }^{37}$ Y.-G. Yoon, P. Delaney, and S. G. Louie, Phys. Rev. B 66, 073407 (2002).

${ }^{38}$ F. Triozon, S. Roche, A. Rubio, and D. Mayou, Phys. Rev. B 69, 121410(R), (2004).

${ }^{39}$ S. Uryu and T. Ando, Phys. Rev. B 72, 245403 (2005).

${ }^{40}$ H. Suzuura and T. Ando, Phys. Rev. Lett. 89, 266603 (2002).

${ }^{41}$ T. Ando, J. Phys. Soc. Jpn. 75, 054701 (2006).

${ }^{42}$ L. Wang, P. S. Davids, A. Saxena, and A. R. Bishop, Phys. Rev. B 46, 7175 (1992).

${ }^{43}$ M. F. Lin and W. K. Kenneth Shung, Phys. Rev. B 47, 6617 (1993).

${ }^{44}$ P. J. Lin-Chung and A. K. Rajagopal, Phys. Rev. B 49, 8454 (1994).

${ }^{45}$ Y. H. Ho, G. W. Ho, S. C. Chen, J. H. Ho, and M. F. Lin, Phys. Rev. B 76, 115422 (2007).

${ }^{46}$ E. A. Taft and H. R. Philipp, Phys. Rev. 138, A197 (1965).

${ }^{47}$ S. Uryu and T. Ando, Phys. Rev. B 74, 155411 (2006).
${ }^{48}$ S. Uryu, H. Ajiki, and T. Ando, Phys. Rev. B 78, 115414 (2008).

${ }^{49}$ Y. Tomio, H. Suzuura, and T. Ando, AIP Conf. Proc. 1399, 815 (2011).

${ }^{50}$ G. Dukovic, F. Wang, D. Song, M. Y. Sfeir, T. F. Heinz, and L. E. Brus, Nano Lett. 5, 2314 (2005).

${ }^{51}$ R. Loudon, Am. J. Phys. 27, 649 (1959).

${ }^{52}$ R. J. Elliot and R. Loudon, J. Phys. Chem. Solids 8, 382 (1959); 15, 196 (1960).

${ }^{53}$ R. Saito, M. Fujita, G. Dresselhaus, and M. S. Dresselhaus, Mater. Sci. Eng. B 19, 185 (1993).

${ }^{54}$ S. Bandow, M. Takizawa, K. Hirahara, M. Yudasaka, and S. Iijima, Chem. Phys. Lett. 337, 48, (2001).

${ }^{55}$ J. Deslippe, C. D. Spataru, D. Prendergast, and S. G. Louie, Nano Lett. 7, 1626 (2007).

${ }^{56}$ S. Uryu and T. Ando, Phys. Rev. B 76, 155434 (2007).

${ }^{57}$ T. Ando, Y. Zheng, and H. Suzuura, J. Phys. Soc. Jpn. 71, 1318 (2002).

${ }^{58}$ R. R. Nair, P. Blake, A. N. Grigorenko, K. S. Novoselov, T. J. Booth, T. Stauber, N. M. R. Peres, and A. K. Geim, Science 320, 1308 (2008).

${ }^{59}$ Z. Q. Li, E. A. Henriksen, Z. Jiang, Z. Hao, M. C. Martin, P. Kim, H. L. Stormer, and D. N. Basov, Nat. Phys. 4, 532 (2008). 